\title{
Das Ich als Geschichte: Erzählte 'Lebensgestalten? Mit einigen Bemerkungen zum Lebenslauff August Hermann Franckes und zur Lebensgeschichte Heinrich Jung-Stillings
}

Das Ich ist narrativ verfasst. Wer wissen möchte, wer er wirklich ist, der muss nur der autobiographischen Geschichte lauschen, die er beständig sich und anderen erzählt. Kohärente Schilderungen des eigenen Lebens stellen kommunikativ Identität(en) her: So oder zumindest so ähnlich lautet die nicht nur von Psychologen, analytischen Philosophen und Historikern diskutierte, sondern auch bei Neurologen und Literaturwissenschaftlern viel beachtete, beliebte These.

Einer ihrer führenden Vertreter, der Neurologe Sacks, interpretiert zum Beispiel den berühmten Fall seines unter dem Korsakow-Syndrom - dem Verlust der Fähigkeit, Erfahrungen, die nach dem Zeitpunkt der Erkrankung liegen, in das autobiographische Gedächtnis zu integrieren - leidenden Patienten Mr. Thompson: Dieser versuchte rast- und ruhelos, die auf ihn einstürmenden Eindrücke um ihn herum in eine kohärente Geschichte zu verweben; mit wenig Erfolg, da er sich nichts davon länger als einige kurze Sekunden merken konnte. Sacks deutet dieses Verhalten als den beinahe verzweifelten Wunsch, eine narrative Kontinuität des gegenwärtigen Erlebens zu sichern, durch die Narration einen festen, mit sich selbst durch die Zeit identischen Bezugspunkt zu schaffen, und so dem eigenen Dasein SSinn zu verleihen (vgl. Sacks 1987).

Ähnlich argumentieren die beiden Psychologen Markowitsch und Welzer. Sie betrachten in ihrem interdisziplinären Ansatz zur Erinnerungsforschung das autobiographische Gedächtnis als ein Phänomen, das uns von allen anderen Primaten unterscheidet: »Es [das autobiographische Gedächtnis bzw. die Autobiographie] integriert das multiple Ich, indem es die wundersame Leistung vollbringt, das Selbst gerade darum als ein immer Gleiches erscheinen zu lassen, weil es sich permanent verändert« (Markowitsch/Welzer 2005). Der Psychologe Bruner meint ebenfalls, dass die Herstellung einer Autobiographie das eigene Selbst, wie auch die eigene Kultur (mit-)konstruiere (vgl. Bruner 2001). McAdams möchte sogar mit seinem »life story model of identity« ein neues Forschungsparadigma begründen (McAdams 2001), und Brockmeier rekonstruiert die Struktur der diskursiven 
Praktiken, die erzählte Identitäten herstellen und kommunizieren (vgl. Brockmeier 2001).

Soviel Einigkeit allerorten ruft freilich auch Kritiker auf den Plan, zumal die Debatte gelegentlich einen gewissen normativen Unterton nicht verhehlen kann. Verfechter der "ethischen Narrativitätsthese« (Strawson [2]) erklären kohärente Selbsterzählungen für moralisch erstrebenswert. Im Extremfall ist ein Leben dann nur so lange ein gelungenes, wie es eine kohärente, in sich geschlossene Lebensgeschichte vorzuweisen hat. Aber nicht nur die ethische Narrativitätsthese ist für den Philosophen Strawson problematisch: Er ist sogar der Ansicht, dass viele Menschen in keiner Weise ihr eigenes Sein als Narration begreifen. Bei ihnen er zählt sich selbst ebenfalls zu dieser Gruppe - handle es sich um sogenannte »Episodiker". Anders als die "Diachroniker« - beide Begriffe schließen sich seiner Argumentation nach gegenseitig aus - haben sie laut Strawson kein Interesse daran, sich als einen durch die Zeit mit sich selbst identisch bleibenden, beständigen Charakter zu konzeptualisieren. Eher empfinden sie ihr Ich als stark in der jeweiligen Gegenwart, im jeweiligen Augenblick verwurzelt, ohne dem besondere Aufmerksamkeit zu schenken, was gestern war oder in einigen Jahren sein wird. Eigene Erlebnisse aus der Vergangenheit werden von ihnen nach Strawson zwar (auch von sinnen`, nicht nur wie durch eine Kamera 'von außen`) erinnert, jedoch hält der Episodiker das das Vergangene erlebende Ich nicht für sich selbst, genauso wenig wie er sich mit dem Individuum identifiziert, das morgen seinen Namen tragen wird. Zwar weiß er freilich um die zeitliche Kontinuität seines biologischen Körpers; eine solche Überzeugung ist für Strawson aber nicht hinreichend dafür, um als Diachroniker zu gelten, geschweige denn, um eine Beschreibung des Ichs als "narrativ« zu rechtfertigen. Das episodische Ich bezeichnet er, um es vom diachronischen Selbst abzugrenzen, als "Selbst* «, und es ist, wie er immer wieder betont, keineswegs in die Form einer Geschichte gefasst (Strawson [2]).

Jens Brockmeier war es, der in diesem Kontext den Begriff der 'Gestalt ins Spiel brachte. Er markiert den Vorgang des autobiographischen Erinnerns, das heißt den Prozess der »reflexiven Identitätskonstruktion « in erzählter Form als »Gestalt in der Zeit" (Brockmeier 2000, 54). Zwar unterscheidet er im Weiteren diverse unterschiedliche "Zeitgestalten" des autobiographischen Erinnerns (von linearen über zyklische, spiralförmige und statische bis hin zu fragmentarischen Gestalten ist alles dabei), jedoch weisen seiner These nach alle autobiographischen "Zeitgestalten« darüber hinaus eine gemeinsame Eigenschaft auf: Die Vergangenheit wird aus der Erzählgegenwart heraus so präsentiert, als habe sie sich zielgerichtet auf eben jenen Augenblick des Erzählens im Hier und Jetzt hin entwickelt; es ist die Rede von »retrospektiver Teleologie«. Damit referiert er auf die Tatsache, dass in einer Autobiographie, idealtypisch betrachtet, der Erzähler meist in einer Art doppelter Ich-Perspektive seine eigene Vergangenheit schildert. Für unsere Zwecke genügt es, hier die einfachste, das ist die lineare, Zeitgestalt in den Blick zu nehmen:»I tell a story about someone who in the course of this story turns out to be me, that is, 
the $I$ who has been telling this story all the time« (Brockmeier 2001, 251). Das letzte Ereignis integriert sozusagen die ganze Autobiographie; sie wird insofern vom Ende her erzählt, und ist vom Anfang der Lektüre an immer 'ganz` (als `Gestalt` sozusagen) da, insofern die Erzählinstanz das Verhalten des Protagonisten meist aus ihrem im Laufe des noch zu schildernden Lebens erworbenen Wissen heraus - entsprechend kommentiert und bewertet. Ganz gemäß der phänomenologischen Tradition der Gestalt-Theorie legt Brockmeier zudem Wert darauf, dass untersuchte Gestalten immer schon erlebte Gestalten, diskursive Praktiken sind.

In diesem Beitrag soll der Versuch unternommen werden, im Anschluss an die Überlegungen Brockmeiers das Gestalt-Konzept aus literaturwissenschaftlicher Sicht sowohl systematisch, als auch an zwei knappen historischen Textbeispielen der Gattung Autobiographie für die Diskussion um die These vom sIch als Geschichter heuristisch fruchtbar zu machen. Wenn Gestalten immer schon erlebte Gestalten bilden, dann wird ihre Untersuchung anhand textueller Phänomene beim historischen Rezipienten ansetzen müssen; Fernziel ist dann eine historische Pragmatik der Gestalt-Wahrnehmung. Um eine dafür taugliche, vorläufige Arbeitsdefinition zu gewinnen bietet es sich an, sich am Klassiker Christian von Ehrenfels zu orientieren: Bekanntlich hat er unter einer "Gestalt« strukturierte Figuren verstanden, die zusätzlich zu ihrer Eigenschaft, stets ‘als Ganzes` wahrgenommen zu werden, durch Übersummativität und Transponierbarkeit als zwei weiteren besonderen "Gestaltqualitäten" auffallen (Ehrenfels 1890).

Im besten Fall vermag dabei ein solchermaßen abstrakter, daher anschlussfähiger und etwas abseits der aktuellen Auseinandersetzungen angesiedelter Begriff als Brücke zwischen unterschiedlichen Disziplinen wie Psychologie, analytischer Philosophie und Narratologie zu dienen. Im schlechteren Fall jedoch erweist er sich mangels empirischer Füllung als schwammig und wenig trennscharf. Um dem entgegenzuarbeiten zielt die Textarbeit im folgenden auch darauf, einige der hier wirkenden narrativen `Gestalt-Mechanismen`mit Mitteln der Textanalyse offenzulegen. Unter Umständen bieten sie eine erste Möglichkeit, die Funktionsweise der erzählten `Lebensgestalten`näher zu charakterisieren. Im kognitionswissenschaftlichen Lager ist der Hinweis gängig, bei der Gestalt-Theorie handele sich um eine rein deskriptive Angelegenheit ohne erklärenden Mehrwert; deshalb soll bei der Textarbeit gleichzeitig auch deutlich werden, was der Gestalt-Begriff in dieser Hinsicht zu leisten vermag.

Wir wollen dies an einem konkreten Beispiel prüfen, bevor wir uns an Johann Heinrich Jung-Stillings fünfbändige Lebensgeschichte, entstanden zwischen 1777 - 1804, heranwagen. Es handelt sich um August Hermann Franckes Lebenslauff, der auf 1690/91 datiert wird. Der Begründer des Halleschen Pietismus - ich gebrauche den Begriff im folgenden lediglich heuristisch mangels einer besseren 
Bezeichnung - schildert darin sein Leben von der Kindheit bis zu seinem 27. Lebensjahr. Das mutet als Zeitpunkt für die Abfassung einer bilanzierenden Autobiographie vielleicht etwas früh an, ist jedoch nicht weiter erstaunlich, wenn man sich vor Augen führt, dass das für Francke wohl zentrale und in gewissem Sinne sein weltliches Leben auch abschließende Ereignis kurz zuvor stattgefunden hatte: Er erlebte seinen sogenannten "Gnadendurchbruch«, es ist die Rede von »Neugeburt«, "Erlösung « und »Bekehrung«. Dem ging ein sündiges, nur äußerlich gottgefälliges Leben voraus, das er aber nach der Rührung durch den Herrn und einem heftigen Bußkampf mit besagter Wiedergeburt endgültig, so glaubt er, hinter sich lassen konnte. In Franckes Leben manifestiert sich so in vorbildlicher Weise die „Ordnung Gottes «: Wer ein ordentlicher Pietist im Sinne Franckes sein will, der muss erst das geschilderte Schema durchlaufen haben, um nicht länger der sündigen Welt zugerechnet zu werden.

Insofern ist hier (i) ein überaus fixes »Handlungsschema " am Werk. Darunter verstehe ich mit Martinez und Scheffel ein aus der Gesamtheit der erzählten Ereignisse abstrahiertes globales Schema der Geschichte, das nicht nur für den einzelnen Text, sondern für ganze Textgruppen (z. B. Gattungen) charakteristisch sein kann (Martinez/Scheffel 2007, 135). Zusammen mit dem eben diskutierten (ii) ,Erzählen von hinten`, mit der »retrospektiven Teleologie« - die vorliegt, da das erzählende Ich nicht müde wird, von Anfang an alles Tun des jungen Francke aus der Sicht des bereits Erweckten als sündig` zu markieren - ergibt das einen recht stark final motivierten Text. Der Lebenslauff wird sozusagen von seinem Ende her verschnürt und zusammengehalten.

Dieser Effekt verstärkt sich noch, wenn man (iii) die in der erzählten Welt waltende Providenz hinzunimmt: Gott ist es, der schon das Kind Francke zum Prediger bestimmt und anschließend sukzessive an der Realisierung seines Plans arbeitet, indem er genau zum richtigen Zeitpunkt Gnade gibt - "Gott ist mir allemahl gleichsam vorgegangen, und hat die klözer und plöcke aus dem wege gehoben" (Francke 1999, 25) - oder merkwürdige `Zufälle` eintreten lässt. Ziel der Bekehrung ist die völlige Hingabe an Gott, das absolute Vertrauen, der freiwillige Verzicht auf den Eigenwillen. In diesem Zustand der »Gelassenheit« ist es dann nicht länger nötig, sich selbst ums "zukünfftige«, welches man sowieso nicht in der Hand habe, zu sorgen (Francke 1999, 21); Gott hatte diese Angelegenheiten immer schon übernommen, und das hat der Bekehrte am Ende des Textes nun auch endlich erkannt: der Text ist per Providenz final motiviert. Nach der abschließenden Bekehrung muss nicht mehr weitererzählt werden, da das (i) Handlungsschema beendet, (ii) der Plot beim aktuellen Ich des Autors in der Schreibgegenwart angelangt, und (iii) Gottes Vorsehung, was Francke betrifft, erfüllt worden ist.

Insofern wird klar, dass für einen zeitgenössischen Leser die hier vorgenommenen Unterscheidungen der diversen Ebenen (i-iii) so wohl nicht offensichtlich gewesen sein dürften. Er hätte eher eine kompakte, durch und durch kohärente Ein- 
heit beobachtet, die das Leben August Hermann Franckes als eines exemplarischen Pietisten vorstellt.

Lässt sich diese kohärente Einheit aber als `Gestaltı, das heißt als `Lebensgestalt‘, charakterisieren? Oben hatte ich mit v. Ehrenfels Übersummativität und Transponierbarkeit als die Definitionsmerkmale der Gestalt benannt. Übersummativität ist wohl gegeben - das Textganze des Lebenslauffs weist Eigenschaften wie große Geschlossenheit und hohe Kohärenz auf, die sich nicht aus der Anhäufung oder Addition seiner Elemente erklären. Erst die Wechselwirkung dieser Elemente, das heißt die Wechselwirkung zwischen jedem Ereignis der erzählten Welt und der finalen Bekehrung (per Handlungsschema, Providenz und retrospektiver Teleologie) schafft den Effekt. Auch transponierbar ist der Text offenbar; so wie man eine als Gestalt identifizierte Melodie auch in anderer als der Original-Tonart sofort wiedererkennt, so war das am Vorbild Franckes geprägte autobiographische Muster (`Ordnung Gottes`, waltende Providenz, retrospektive Teleologie) wohl auch auf die Lebensläufe anderer Menschen übertragbar (von Transponierbarkeit kann man immer dann sprechen, wenn die Gestalt auch nach dem Austausch aller Elemente noch erkennbar bleibt): Francke zumindest schien davon auszugehen, wenn er 1692 an Philipp Jakob Spener schrieb,

Wegen des jüngst uns zugesandten Briefes eines mit dem Atheismo luctierenden Menschen sende hierbey den Anfang und Fortgang meiner Bekehrung, weil die Exempel mehr zu movieren pflegen und gewiss eben desgleichen in meinem Gemüth vorgegangen. Könnte solches, so es rathsam befunden wird, quamquam nomine meo plano suppresso, communiciret werden. (Francke 1999, 79)

Gary Sattler ist zuzustimmen: »In Francke one sees the story of the Pietist everyperson.« (Sattler 1989, 1). Man könnte auch, etwas präziser, sagen: In Franckes Autobiographie erkennt jedermann sofort die Lebensgestalt eines mustergültigen Pietisten.

\section{3.}

An dieser Stelle machen wir den angekündigten Sprung zur fünfbändigen Autobiographie Heinrich Jung-Stillings; ist hier ebenfalls eine Lebensgestalt zu beobachten?

Im Jahr 1777 erschien anonym, von Goethe ohne Wissen des Autors zum Druck befördert, der erste Band der Autobiographie Heinrich Jung-Stillings (1740-1817): Henrich Stillings Jugend. Eine wahrhafte Geschichte rief eine so große Resonanz hervor, dass bereits im nächsten Jahr die Jünglings-Jahre und die Wanderschaft vorgestellt wurden. 1789 folgten das Häusliche Leben und 1804 schließlich die Lehr-Jahre. Das 1817 von Jung-Stillings Enkel Wilhelm Schwarz aus dem Nachlass publizierte Fragment Heinrich Stillings Alter komplettierte die Lebensgeschichte. Wir lernen Jung-Stilling als Sohn eines streng gläubigen Dorf- 
schullehrers und Schneiders kennen, der sein Auskommen zunächst unter anderem als Hauslehrer und Kaufmannsgehilfe sucht, um dann völlig mittellos, allein auf Gott vertrauend, in Strassbourg ein Medizinstudium zu beginnen und anschließend als Augenarzt zu praktizieren. Dem Ruf auf eine Professur für Kameralwissenschaften folgt schließlich seine Anstellung als Berater des Kurfürsten Karl Friedrich, so dass er ungehindert als Erbauungsschriftsteller publizieren kann.

Es stellt sich die Frage, ob hier ebenfalls eine kohärente Lebensgestalt wie die von August Hermann Francke vorliegt. Auffällig ist, dass zwar beide Texte auf die Erbauung ihrer Leser abzielen - Jung-Stilling spart nicht mit erbaulichen Kommentaren und wird auch nicht müde, seine Leser stets an die wirkende Providenz Gottes zu erinnern -, sich jedoch der Eindruck der Kohärenz und Geschlossenheit, das heißt der Gestalt-Haftigkeit, bei dem neueren Text nicht mehr mit derselben Selbstverständlichkeit einstellen mag. Es steht zu vermuten, dass Übersummativität und Transponierbarkeit gelitten haben. Das ist im Detail zu prüfen:

Franckes Lebenslauff zeichnete sich noch durch eine dreifache Teleologie aus, was Übersummativität bewirkte: Sowohl das (i) Handlungsschema aus Rührung - Bußkampf - Bekehrung, wie auch das (ii) Muster der retrospektiven Teleologie und die (iii) in der erzählten Welt wirkende Providenz fielen am Ende der Autobiographie im Erweckungserlebnis in eins zusammen. Auf diese Weise sorgten sie für eine Kohärenz des Ganzen, die die bloße aufaddierende Aneinanderreihung der Einzelereignisse in keiner Weise zustande gebracht hätte. Wie steht es nun um diese drei, ich nenne es 'Gestalt-Mechanismen`, im Text Jung-Stillings?

Beginnen wir mit dem (i) Handlungsschema: In Jung-Stillings Geschichte ist zumindest das Handlungsschema von sündigem Leben, Rührung, Bußkampf und Gnadendurchbruch nicht mehr gegeben; es ist nicht mehr prominent für die Kohärenz des Ganzen funktional wirksam. Hier sorgt nicht ein einzelnes Wiedergeburtserlebnis für die finale Integration des Textes; stattdessen finden sich einzelne Elemente des Handlungsschemas verteilt in der ganzen Autobiographie wieder. Gemeinsam ist diesen Einsprengseln das entsprechende Vokabular, die permanente Berufung auf göttliche Providenz und - das ist neu - der ständige Bezug zu Beruflichem. Letzteres spielte auch schon bei Francke eine entscheidende Rolle, ist notwendiger Bestandteil des gelungenen pietistischen Werdegangs. Trotzdem lag dort das Schwergewicht doch noch eher auf den Umbrüchen im `Inneren agonisten, seine weltliche Profession hatte dem lediglich zu entsprechen und Ausdruck des gottesfürchtigen Seelenlebens zu sein (Francke wird ja auch folgerichtig Prediger). Jetzt indessen wird die berufliche Laufbahn selbst zum Hauptgegenstand der Lebensgeschichte; man könnte von stypologischer Säkularisation` (Niggl) der pietistischen Autobiographie oder besser von einer Schwerpunktverlagerung

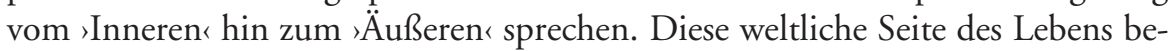
steht für die Hauptfigur aus so vielen verschiedenen Berufen wie Schulmeister und Schneider, Kaufmannsgehilfe, Augenarzt oder Professor, was die Einhaltung des mit einem und nur einem finalen Telos ausgestatteten diskutierten Handlungs- 
schemas so gut wie unmöglich macht, da es an einem endgültigen und eindeutig als solchen identifizierbaren Endpunkt fehlt.

Allerdings entspricht die zeitliche Anordnung der Tätigkeiten, was den mit ihnen jeweils verbundenen gesellschaftlichen Status angeht, einer aufsteigenden Linie. Insofern wird es möglich, nach Wegfall des den Text integrierenden Schemas wenigstens die dazugehörige (iii) Providenz zu erhalten, die nun auf die Geschichte vom geglückten sozialen Aufstieg übertragen werden kann, und so für eine gewisse Geschlossenheit zu sorgen vermag; die grundsätzlich positive Entwicklungstendenz ist dafür wohl von entscheidender Bedeutung. Sollte die Integration des Geschehens per göttlicher Vorsehung funktionieren, so ist zu erwarten, dass der Schluss der Selbstbiographie uns einen Stilling vorstellt, der seine Bestimmung - den ihm von Gott immer schon zugedachten Beruf - endlich gefunden hat. In diesem Fall wäre alles wieder in der schönen Ordnung, entspräche also wieder der guten Gestalt, die noch die Autobiographie Franckes prägt. Auf diese Weise könnte dann aus einem "fragmentarischen Leben ein Ganzes" gemacht werden (Hirzel 1998, 219).

Wie genau versucht nun der Verfasser die Lebensgeschichte final zu motivieren und dadurch die 'gute`, da kohärente und abgeschlossene Gestalt zu aktualisieren? Er verknüpft die einzelnen Elemente weniger mit dem jeweils vorausgehenden bzw. nachfolgenden, sondern er bezieht jedes Ereignis direkt auf den Schlusspunkt des Textes, also auf das Erreichen der von Gott für den Helden vorgesehenen Profession. Wie bei der Biographie Franckes zeigt sich diese Formation am deutlichsten an den Wendepunkten des Geschehens; das wären in unserem Fall vor allem, aber nicht nur, die zahlreichen Berufswechsel Stillings. An solchen `Turningpoints insbesondere dürfte die permanent hinter dem Erzählten waltende Providenz aufscheinen. Bei ihrer Diskussion sollte sichtbar werden wird, wie schwierig es ist, vom dritten Gestalt-Mechanismus, der Providenz, bei der Erzählung einer säkularen Karriere zu retten, was zu retten ist:

Einer der Berufswechsel geschieht 1768, als sich Stilling, wie in der Wanderschaft berichtet wird, plötzlich zum Arzt berufen fühlt. Der Anstoß dazu kommt von außen, während er selbst eher passiv bleibt. Sein aktueller Arbeitgeber Herr Spanier, bei dem er als Hauslehrer und Kaufmannsgehilfe beschäftigt ist, verkündet nauf einmahl« er wisse nun, was aus seinem Bediensteten werden solle: »Hört Präceptor! fieng endlich Spanier an: mir fällt da auf einmahl ein, was Ihr thun sollt, Ihr müsst Medicin studieren«", worauf dieser sofort erklärt:

das ists, wozu ich bestimmt bin. Ja, ich fühl in meiner Seelen, das ist das grosse Ding, das immer vor mir verborgen gewesen, das ich so lange gesucht, und nicht habe finden können! Dazu hat mich der himmlische Vater von Jugend auf durch schwere und scharfe Prüfungen vorbereiten wollen. Gelobet sey der barmherzige Gott, dass er mir doch endlich seinen Willen offenbaret hat, nun will ich auch getrost seinem Wink folgen. (Jung-Stilling 1976, 238) 
Anschließend rekonstruiert er, inwiefern sein ganzes bisheriges Leben auf das scheinbar jetzt endlich entdeckte Ziel hin von Gott aufs Beste eingerichtet gewesen sei (vgl. Jung-Stilling, 238 f.). In der augenblicklichen Situation hält er die Medizin für das, was von allem Anfang an für ihn vorgesehen war und verknüpft deshalb alles bis jetzt Geschehene final durch sein neu gewonnenes Telos. Die wichtige Frage, ob er selbst als Mitverursacher der Veränderung gelten kann, beantwortet der Text hier recht eindeutig; zusätzlich zum für die Passivität Stillings sprechenden Anstoß von außen geht dem dargestellten Wortwechsel zwischen Spanier und Stilling ein merkwürdiges Erlebnis des letzteren voraus: Henrich sitzt beim "Coffeetrinken am Tisch", als ihm Reitz' Historie der Wiedergebohrnen in die Hände fällt. "[E]r blätterte ein wenig darinnen herum ohne Absicht und ohne Nachdenken" und stößt auf die Geschichte eines Mannes, der die griechische Sprache lernt und bei dem Wort »Eilikrinea - was soviel wie `Offenbarung heißt - ein »vortrefliches Gefühl « hat. Stilling geht es auf der Stelle ganz genauso, er »fühlte [...] einen unwiderstehlichen Trieb in sich" es dem Mann gleichzutun und spürt einen »starken Zug zu etwas, das er noch gar nicht kannte, auch nicht zu sagen wusste, was es war" (Jung-Stilling, 236 f.), um dann wenig später seine vermeintlich finale Bestimmung zum Arzt zu erkennen; der aktive Part bei Schicksalswendungen bleibt offensichtlich Gott vorbehalten. Seine Macht ist es, die aus der Sicht des Helden wie hier auch des Erzählers die Fäden zieht. An dieser These wird der Protagonist den gesamten verbleibenden Rest seines Lebens über eisern festhalten. Dagegen scheint er schon wenige Jahre später von der Arzneikunde als seiner endgültigen Bestimmung keineswegs mehr so vollauf überzeugt zu sein. Nach und nach entwickelt sich sein "tiefe[r] Widerwillen" (Jung-Stilling, 303) zu einem ausgewachsenen Konflikt: "Jetzt fing er an zu zweifeln, daß ihn Gott zur Medizin berufen habe; er fürchtete, er habe denn doch vielleicht seinem eigenen Triebe gefolgt und werde sich nun lebenslang mit einem Beruf schleppen müssen, der ihm äußerst zuwider sey« (JungStilling, 337). Schließlich findet Stilling eine neue "ganze Bestimmung " Jung-Stilling, 354), wiederum durch einen Impuls von außen - diesmal ist es ein Brief von Eisenhart, der ihm einen Lehrstuhl für "Landwirthschaft, Technologie, Handlung und Vieharzeneykunde " andient - und auch diesmal angeblich ohne irgendwelches eigenes Zutun. Noch einmal deutet er sein ganzes Leben auf die gerade entdeckte Berufung hin, nur zielt er jetzt statt auf die Medizin auf den Beruf des Professors (vgl. Jung-Stilling, 354 ff.). Dass jene Offenbarung des göttlichen Plans ebenso wie ihre Vorgänger wird revidiert werden müssen, und zwar zu Gunsten der Erbauungsschriftstellerei, kann kaum noch überraschen. Stilling ist also eine Figur, die sich meist zu Beginn des jeweiligen Bandes an das eine, nun erreichte Ziel Gottes glaubend zeigt, nur um dann ins Zweifeln zu geraten und am Ende von einer immer anderen, seiner neuen 'wahren Berufung überzeugt zu sein.

Wie verhält sich dazu die Perspektive des Erzählers, das heißt wie steht es um den verbleibenden Gestalt-Mechanismus der (ii) retrospektiven Teleologie? Letztendlich geht es dem Erzähler nicht besser als seinem Personal: Für sämtliche Berufs- 
wechsel Stillings lässt sich zeigen, wie der Erzähler seine Einschätzung ein- und desselben Ereignisses als ,finale Bestimmung` oder aber als 'von sündigem Eigenwillen verursacht $<$ je nach Bedarf und seiner momentanen Erzählposition im Fortgang des Lebenslaufs entsprechend variiert. Was der Sprecher im ersten Band verkündet, das muss im letzten nicht mehr unbedingt Gültigkeit haben; bei Francke wäre eine solche Unsicherheit undenkbar gewesen. Die Finalität einer von Gott gegebenen Position scheint nicht länger mit Gewissheit feststellbar zu sein, weder für die Figur, noch für die Erzählinstanz; die Autobiographie wird nicht wirklich länger von ihrem Ende her erzählt: Statt dessen geht das Leben immer weiter. `Motivation von hinten sieht sich vor das bei Francke noch unbekannte Problem gestellt, ein ihr Wirken abschließendes Geschehnis überzeugend als solches zu markieren.

Ganz ähnliche Schwierigkeiten wie die mehrfachen Berufswechsel bringen ebenso die drei im Laufe der Zeit von Stilling eingegangenen Ehen mit sich; hier ist exakt derselbe Mechanismus am Werke. Auch in diesem Bereich scheint Jung-Stilling je nach seiner aktuellen Position in der fortlaufenden Geschichte das eine Mal der Meinung zu sein, die einzelne Heirat sei wirklich Folge seines 'gelassenen` Vertrauens auf Gottes Führung, während er das andere Mal seine eigenen Entscheidungen bzw. Neigungen zumindest als Mitverursacher in Erwägung zieht.

Die so in die erzählte Welt kommenden Fehldeutungen des Helden bzw. Erzählers müssen im Fortgang der Geschichte freilich sgeheilt‘ werden. Erwartungsgemäß wird die Vorsehung verantwortlich gemacht. Sie habe Stilling z. B. dazu gebracht, den falschen Beruf für seine wahre Berufung zu halten, so dass er nun in der falschen Profession tüchtig zu leiden hat. Das sei typisch für die

Methode der Vorsehung [...]: Stilling war mit Leidenschaft und unreiner Begierde dem Ziel entgegen gelaufen, es hatte sich Stolz, Eitelkeit, und wer weiss nicht was alles, mit eingemischt [...]. Es ist die Maxime der ewigen Liebe, dass sie ihre Zöglinge geschmeidig und ganz in ihrem Willen gelassen macht, ehe sie weiter geht. (Jung-Stilling, 358)

Sie läutert ihre Schützlinge, um deren Makel auszumerzen. "Läuterung « - das ist der semantische Kitt, mit dem Jung-Stilling die Risse in der Gestalt seiner Lebensgeschichte wenigstens provisorisch zu verschließen sucht.

Für Stilling sind die Hingabe an die Vorsehung und ein verantworteter Gebrauch der eigenen Vernunft, außer zur Übergabe des eigenen Willens an Gott, nur schwer vereinbar. Das Thema bestimmt dann auch den ganzen »Rückblick auf Stillings bisherige Lebensgeschichte", der sich daran abarbeitet, diese als "Geschichte der Vorsehung in seiner [Stillings] Führung" (Jung-Stilling, 437) und somit als final motivierte Einheit mit dem Telos `Erbauungsschriftsteller zu präsentieren - die Kohärenz und die sgute Gestalt ‘ des Textes verstehen sich nicht mehr von selbst, sie müssen eigens noch einmal in einem abschließenden Überblick her(aus)gestellt werden; im Prinzip sieht sich die Autobiographie demnach hier mit demselben Problem konfrontiert, das auch schon die Darstellung der zahlreichen Berufswechsel und Hochzeiten Stillings prägte. 
Wie Albrecht Willert bemerkt: Jung-Stilling hat sich zu sehr aufs Denken eingelassen, seine Kalkulation geht nicht wirklich auf (vgl. Willert 1982, 87). So dokumentiert das aus dem Nachlass publizierte Fragment Heinrich Stillings Alter erneute Zweifel an der Endgültigkeit der Berufung:

Du hast nie einen Schritt gethan, dich eigenmächtig aus der Lage zu setzen, in die dich die Vorsehung geführt hatte; [...] Ist dies aber auch jetzt der Fall? - Hast Du weder mittelbar noch unmittelbar dazu beygetragen, dass Dich der Kurfürst von Baden hierher berufen hat? War Dein Grundtrieb, für den Herrn und sein Reich zu wirken, rein? Lag nicht in der Tiefe deiner Seele auch die Eitelkeit verborgen, als ein grosses Licht in der Kirche Gottes zu glänzen, und durch deine Schriften in aller Welt berühmt zu werden? (Jung-Stilling, $632 \mathrm{f}$.)

Der im »Rückblick« erreichte Stand an Gewissheit, was das Anlangen am finalen Punkt betrifft, war wie schon seine am jeweiligen Ende der übrigen Bände platzierten Vorgänger nicht von Dauer. Alle Abschlussversuche scheitern mehr oder weniger an ihrer prinzipiellen Revidierbarkeit, wobei die Revision in der Zukunft zumindest möglich, wenn nicht sogar aller Erfahrung und Vernunft aus der Lebensgeschichte nach wahrscheinlich ist. Die Installation eines unüberschreitbaren Telos am Ende der Autobiographie muss unter solchen Umständen eigentlich fehlschlagen, das `Auf-Dauer-Stellen` bleibt ein Versuch. In der Lebenswelt Stillings kann man bereits, anders als noch bei Francke, durch die Entwicklung immer eines Besseren belehrt werden - der Gestalt-Mechanismus (iii) ,finale Motivation durch Providenz ist damit, genau wie zuvor bereits das (i) pietistische Handlungsschema und die (ii) retrospektive Teleologie, mangels eines wirklich stabilen Ziels problematisch geworden. Anstatt sich von selbst zu verstehen, muss die Gestalt nun vom Erzähler mühsam immer wieder von neuem etabliert werden. Vornehmlich geschieht das durch die Idee von der "Läuterung « unter nicht unerheblichem semantischem Aufwand. Von der Übersummativität eines August Hermann Francke ist nicht mehr viel übrig. Wie sieht es aber mit der Transponierbarkeit der Lebensgestalt Jung-Stillings aus? Ließe sich die in der Lebensgeschichte mühsam konstruierte Lebensgestalt wenigstens prinzipiell auf ein anderes Individuum übertragen?

Ich denke, dies ist recht eindeutig nicht der Fall; zu einzigartig sind bereits die Ereignisse, denen sich Jung-Stilling ausgesetzt sieht. Sein Text taugt nicht mehr zum erbaulichen Exempel, wenigstens nicht zu einem, das man im Streben nach einem gottgefälligen Leben eins zu eins nachahmen könnte; die Lebensgeschichte gibt keine auf andere Personen transponierbare Gestalt mehr ab.

Was aber durchkreuzt immer wieder die Bemühungen um Ganzheit, um eine kohärente Gestalt? Insgesamt kann man wohl sagen, dass das Problem gar nicht so sehr in den zahlreichen Krisen besteht, sondern eher im Fehlen eines fixen Endpunktes. Und dieser wiederum ist verloren gegangen, weil Jung-Stilling sich einem modernen, einzigartigen sozialen Aufstieg ausgesetzt sieht; seine "Karriere" im Sinne Luhmanns ist ein Musterbeispiel für ein aus der sich nun nach und nach funktional ausdifferenzierenden Gesellschaft exkludiertes Individuum. Um ein ganzes Ich darzustellen - etwa in einer Autobiographie - bedarf es jetzt der Kon- 
zentration auf die Zeitdimension des beobachteten Lebens. Die teleologische Lebensgestalt kann ihre Wirkung nicht mehr entfalten, da sich der erzählte Werdegang selbst, wie oben gezeigt wurde, der Herstellung von Übersummativität durch Teleologie beinahe schon systematisch widersetzt.

Freilich bleibt es nicht dabei; in den nächsten Jahrzehnten wird sich, sozusagen als Konkurrenzmodell, eine Individualitätssemantik der Perfektibilität etablieren, die voll und ganz auf Entwicklung in der Zeit setzt. Jeder hat sich dann zu bilden, immer weiter hinein in eine bessere, nach hinten unabgeschlossene persönliche $\mathrm{Zu}$ kunft. Die Einzelereignisse des jeweiligen Lebenslauf werden nun nicht mehr mit einem fixen Endpunkt, sondern jeweils mit dem zeitlich davorliegenden und direkt nachfolgenden Geschehen kausal verknüpft. Spätestens hier hat dann die verschriftlichte Autobiographie die Aufgabe übernommen, das multiple Ich zu integrieren, indem sie das Selbst gerade darum als ein immer Gleiches erscheinen lässt, weil es sich permanent verändert (vgl. Markowitsch/Welzer 2005). Entlang der auf dieses neue Bezugsproblem ausgerichteten Gestalt der >Perfektibilität‘ bzw. `Bildung‘ lässt sich das mehr oder weniger komfortabel bewältigen. Hat man diese Gestalt dagegen noch gar nicht im Reportoire bzw. klammert sich mehr oder weniger fest an die teleologische Gestalt aus der pietistischen Tradition, so bekommt man als Absolvent eines sozialen Aufstiegs voller Kontingenzerlebnisse unweigerlich genau die Schwierigkeiten, mit denen sich Heinrich Jung-Stilling bei der Abfassung seines Lebenslauf immer wieder konfrontiert findet, sobald perfektibler Inhalt und teleologische Gestalt in Konkurrenz zueinander treten.

Ist demnach also das Gestalt-Konzept von Nutzen zur Beantwortung historischer Fragestellungen im Rahmen der Debatte um »Das Ich als Geschichte«? Erbringt es im Vergleich mit 'herkömmlichen Verfahren der Textanalyse einen Mehrwert an Erkenntnissen? Als ’herkömmlicher Verfahren wären bei der Auseinandersetzung mit der Lebensgeschichte Jung-Stillings die Begriffe ‘finale Motivation` und vor allem 'Kohärenz in Frage gekommen. Abgesehen davon, dass die Rede von der 'Kohärenz eines Textes selbst alles andere als klar ist, steht doch die Bezeichnung laut Reallexikon »zumeist eher ungenau für eine gewisse Geschlossenheit von Texten" (Stuck 2000, 281): Hat das Gestalt-Konzept, verstanden als Zusammenspiel von Übersummativität und Transponierbarkeit, demgegenüber einen Vorteil zu bieten? Nicht von der Hand zu weisen ist wohl die große Anschaulichkeit des Beschreibungsbegriffs , Gestaltı. Er macht es einfacher, bestimmte Textkonstellationen zu beobachten und diese Beobachtungen zu kommunizieren: So wird deutlicher als beim Gebrauch der Termini `Kohärenz` und/oder 'Geschlossenheit`, dass ein als `Gestalt ‘ erscheinender Text nicht nur stimmig oder schlüssig ist, sondern darüber hinaus auch z. B. als Ganzes genommen einen bestimmten 'Sinn ‘ transpor- 
tieren kann, der nicht auf die Summe seiner Teile zu reduzieren ist (,Übersummativität`). Dieser Sinn kommt einer Autobiographie insofern entgegen, als autobiographische Texte im Allgemeinen auch identitätsstiftende Texte sind; sie aktualisieren die Geschichte, die sich jede Person über sich selbst erzählt. Ein ständig mitgeführter `Sinngehalt $\_-$z. B. die in der erzählten Welt wirkende Providenz - bietet sich als mitlaufender, stabiler Referenzpunkt an; er garantiert, dass man heute derselbe ist, der man gestern war und morgen auch noch immer sein wird. Das ist mehr als bloße Kohärenz und lässt sich besser als `Gestalt‘ umschreiben.

Zudem konnten in der konkreten Textarbeit (i) pietistisches Handlungsschema, (ii) retrospektive Teleologie und (iii) die in der Diegese wirkende Providenz als Mechanismen markiert werden, die die Autobiographie integrieren und sverschnüren`. Weiter oben wurden sie bereits als `Gestalt-Mechanismen ‘ ausgewiesen; die narratologischen Werkzeuge ermöglichen es, die genauere Funktionsweise narrativ verfasster Lebensgestalten näher zu erläutern und damit dem oft mit Vorwürfen der ,Schwammigkeit konfrontierten Gestalt-Begriff in dieser Hinsicht etwas aufzuhelfen. Je nachdem, in welcher Weise die einzelnen Ereignisse im Text miteinander verknüpft werden, entsteht eine andere 'personaler Gestalt: Wird jedes Einzelereignis mit dem zeitlich letzten Geschehnis in der erzählten Welt verbunden, so ergibt sich eine stark teleologisch wirkende Gestalt; stellt man dagegen Zusammenhänge jeweils zwischen einem Einzelereignis und in der Zeitfolge direkt vorangehenden bzw. nachfolgenden Geschehnissen her, so arbeitet der Text eher auf eine Rezeption als von `Bildung` und >Perfektibilität` geprägtes Ich hin. Übersummativität und Transponierbarkeit (man denke nur an den Erfolg der Idee vom Bildungsroman) sind in beiden Fällen gegeben. Andere, weitere Gestalt-Konstellationen sind freilich denkbar - es sei nur an die diversen "Zeitgestalten « in den von Brockmeier untersuchten autobiographischen Narrationen erinnert.

Es bleibt die nach wie vor offene Fragen nach der Dienlichkeit des Gestalt-Konzepts für die systematische Diskussion der Leitthese vom narrativ verfassten Ich. Die oben bereits referierten Positionen ihrer Fürsprecher treffen auf die Argumentation Strawsons als einem ihrer Hauptgegner. Er unterscheidet zwischen »Episodikern" und "Diachronikern " - man kann seiner Ansicht nach nur das eine oder andere von sich behaupten, jedoch niemals beides zugleich - und geht davon aus, dass das "Selbst* « der ersteren strikt in der aktuellen Gegenwart verwurzelt bleibt, ohne Bezüge zu früheren oder zukünftigen Selbst*-Versionen herzustellen, währende letztere dazu tendieren, sich selbst als einen durch die Zeit mit sich identischen Charakter zu vergegenständlichen. Stephan Packard hat darauf hingewiesen, wie auffallend die Differenzierung zwischen episodischen und diachronen Personen einer Literaturwissenschaftlern wohlbekannten narrativen Spannung ähnelt (vgl. Packard [1]): Gerade in autobiographischen Erzählungen kommt es typischerweise zu einem Nebeneinander von Erzählinstanz und Figurenperspektive, von "Kontinuum" des erzählenden und jeweiliger aktueller "Situation" des als Figur die eigene Geschichte erlebenden Selbst: "I tell a story about someone who in 
the course of this story turns out to be $m e$, that is, the $I$ who has been telling this story all the time" (Brockmeier 2001, 251). Im Normalfall scheinen sich diachrone $(\rightarrow$ Erzählinstanz) und episodische ( $\rightarrow$ Figurenperspektive) Kognitionen insofern nicht auszuschließen, sondern eher gemeinsam aufzutreten, und zwar in narrativen Formationen. Letztendlich wird ein Selbst* lediglich vor dem Hintergrund eines diachronen Ichs überhaupt denkbar, die Alternative muss bewusst gehalten werden (vgl. Packard [1]). Ohne seinen Gegenbegriff, das heißt ohne die Vorstellung vom diachronen Ich als Folie zu benutzen, bleibt das Selbst* schlicht und einfach unsichtbar: Wenn jemand von sich behauptet, er sei nicht mit sich selbst in der Vergangenheit oder Zukunft identisch und lebe statt dessen voll und ganz im aktuellen Augenblick des Hier und Jetzt - nenne also ein Selbst* sein eigen - so kann er das nur tun, indem er aktiv die Existenz seines herkömmlichen, durch die Zeit mich sich identischen, Selbst verneint.

Beobachtet man diesen Sachverhalt mithilfe des gerade explizierten GestaltKonzepts, so wird deutlich, warum das so ist, und es zeigt sich, welcher Gestalt-Mechanismus hier am Werk ist: Anstatt Einzelereignisse des Geschehens miteinander oder mit dem letzten Ereignis des Textganzen zusammenzufügen, wird im Fall des episodischen Ichs der Verknüpfung zwischen jeder Einzelepisode und den anderen Einzelepisoden bzw. der Verbindung zwischen Einzelepisode und der Gesamtgeschichte vom Ich sozusagen absichtlich und ganz bewusst der Wert Null zugewiesen. Anschaulich gesagt liegt quasi die Umkehrung der Gestalt-Bemühungen vor, wie sie sich in der Autobiographie Jung-Stillings gezeigt hatten: Während dort der Text sich daran abarbeitet, Verknüpfungen der Einzelereignisse der Lebensgeschichte mit dem Textganzen herzustellen, jedoch immer wieder scheitert, somit also keine gute Gestalt hergestellt werden kann, versucht ein episodisches Selbst sozusagen jene Verknüpfungen mit aller Macht zu ignorieren und sie als nicht existent auszuweisen. Ganz ohne Geschlossenheits- und Kohärenzbestrebungen oder, noch genauer, ohne Gestaltbildung kann man insofern wohl überhaupt kein Selbst, auch kein Selbst* denken - man muss sich mindestens die Mühe machen, die Gestaltformation aktiv zu negieren. Die Gestaltmechanismen tun derweil dessen ungeachtet ihre Arbeit.

\author{
Steffanie Metzger \\ Institut für deutsche Philologie \\ Ludwig-Maximilians-Universität München
}




\section{Literatur}

Jens Brockmeier, Autobiographical time, Narrative Inquiry 10:1 (2000), 51-73.

-, From the end to the beginning. Retrospective teleology in autobiography, in: Jerome Bruner/Donal Carbaugh (Hg.), Narrative and Identity. Studies in Autobiography, Self and Culture, Amsterdam/Philadelphia 2001, 247-280.

Jerome Bruner, Self-making and world-making, in: Jerome Bruner/Donal Carbaugh (Hg.), Narrative and Identity. Studies in Autobiography, Selfand Culture, Amsterdam/Philadelphia 2001, 25-38.

Christian von Ehrenfels, Über Gestaltqualitäten, Vierteljahresschrift für wissenschaftliche Philosophie 4 (1890), 249-292.

August Hermann Francke, Herrn M. August Hermann Franckens vormahls Diaconi zu Errfurt [...] Lebenslauff, in: Lebensläufe August Hermann Franckes, hg. v. Markus Matthias, Leipzig 1999, 5-32.

Martin Hirzel, Lebensgeschichte als Verkündigung. Johann Heinrich Jung-Stilling - Ami BostJohann Arnold Kanne, Göttingen 1998.

Johann Heinrich Jung-Stilling, Lebensgeschichte, hg. v. Gustav Adolf Benrath, Darmstadt 1976.

Hans Markowitsch/Harald Welzer, Das autobiographische Gedächtnis. Hirnorganische Grundlagen und biosoziale Entwicklung, Stuttgart 2005.

Matias Martinez/Michael Scheffel, Einführung in die Erzähltheorie, München ${ }^{7} 2007$.

Dan P. McAdams, The psychology of life stories, Review of General Psychology 5:2 (2001), $100-122$.

Oliver Sacks, Der Mann, der seine Frau mit einem Hut verwechselte, Reinbek 1987.

Gary Sattler, Nobler than the Angels, lower than a Worm. The Pietist View of the Individual in the Writings of Heinrich Müller and August Hermann Francke, Lanham/New York/London 1989.

Elisabeth Stuck, Art. `Kohärenzı, in: Braungart et al. (Hg.), Reallexikon der Literaturwissenschaft. Bd. 2, Berlin, New York 2000, 80-81.

Albrecht Willert, Religiöse Existenz und literarische Produktion. Jung-Stillings Autobiographie und seine frühen Romane, Frankfurt am Main/Berlin 1982.

[1] Stephan Packard: Jede Narration ist Dihärese. Eine Reaktion auf Galen Strawson, parapluie 24 (2007/08): Wildwüchsige Autobiographien. < http://parapluie.de/archiv/auto biographien/narrationen/> (29.5.2008).

[2] Galen Strawson, Ein Irrtum unserer Zeit. Nicht jedes Leben ist narrativ, parapluie 24 (2007/08): Wildwüchsige Autobiographien. <http://parapluie.de/archiv/autobiographi en/episoden/> (29.5.2008) (= A Fallacy of Our Age: Not Every Life is a Narrative, Times Literary Supplement, October 15, 2004, 13). 